\title{
Receiver Operator Characteristics (ROC) Analysis of Real-Time Shear Wave Elastography Information Health Monitoring in Liver Fibrosis with Chronic Hepatitis B Virus Infection
}

\author{
Zhexia Zhao $\mathbb{D}^{\mathbb{D}}$, Xiaoyan Chen $\left(\mathbb{D}\right.$, Yanxia Zhang $\mathbb{D}^{\mathbb{D}}$, Qian Song $\mathbb{D}$, Jiping Xue $\mathbb{D}$, \\ and Chunsong Kang iD \\ Department of Ultrasound, Shanxi Bethune Hospital Shanxi Academy of Medical Sciences, Taiyuan City 030032, China \\ Correspondence should be addressed to Zhexia Zhao; zhaozhexia@a.sxmu.edu.cn
}

Received 6 April 2021; Revised 3 June 2021; Accepted 22 June 2021; Published 5 July 2021

Academic Editor: Gustavo Ramirez; drgustavophd@gmail.com

Copyright (c) 2021 Zhexia Zhao et al. This is an open access article distributed under the Creative Commons Attribution License, which permits unrestricted use, distribution, and reproduction in any medium, provided the original work is properly cited.

To investigate the effectiveness of shear-wave elastography (SWE) based on e-health in diagnosing liver fibrosis in patients infected with hepatitis B virus (HBV) and provide theoretical basis for early diagnosis and treatment of patients with chronic HBV infection, in this study, 73 patients with chronic HBV infection who underwent e-health SWE examination were divided into chronic hepatitis B group (51 cases) and hepatitis B cirrhosis group (22 cases), and another 20 healthy volunteers were introduced as control. The contents of second liver two half-and-half, hyaluronic acid (HA) of liver fibrosis, laminin (LN), pAUVollagen III N terminal peptide (PIII NP), collage type IV (CIV), and HBV-DNA were detected. SWE was used to measure the elastic modulus in the S2 and S3 segments of the left outer lobe of the liver, S4 segment of the left inner lobe of the liver, S5 and S8 segments of the right anterior lobe of the liver, and S6 and S7 segments of the right posterior lobe of the liver. Finally, the correlations between the four examination results of liver fibrosis and the elastic modulus values were compared, and the receiver operator characteristic curve (ROC) for SWE technical diagnosis was drawn. The results showed that there was no significant difference in HBV-DNA content between the two groups $(P>0.05)$; the contents of alanine transaminase (ALT) and aspartate aminotransferase (AST) in the blood of chronic hepatitis B group were significantly higher than those in the liver cirrhosis group $(P<0.05)$; HA, PIII NP, and CIV contents were significantly lower than the liver cirrhosis group $(P<0.05)$; the success rates of SWE detection in S5 and S6 segments of the two groups were 100.00\%, and the elastic modulus of the hepatitis B cirrhosis group was significantly higher than that of the chronic hepatitis B group $(P<0.05)$; correlation results showed HA, PIII NP, and CIV of two groups of patients and elastic modulus value in S5 and S6 segments showed positive correlation $(P<0.05)$; ROC curve analysis showed that AUC (area under the curve) was 0.866 , the sensitivity was $89.59 \%$, and the specificity was $76.49 \%$, which indicated that the liver elastic modulus value measured by e-health SWE technology can quantitatively show liver fibrosis in patients with chronic $\mathrm{HBV}$ infection, and its application in the diagnosis of clinical liver fibrosis was real and feasible.

\section{Introduction}

$\mathrm{HBV}$ is the pathogen that causes hepatitis B. When people get infection of HBV, various acute and chronic hepatitis $b$ occur to them that will lead to the occurrence of liver fibrosis and posing a serious threat to their health and quality of life [1]. Liver fibrosis is a pathophysiological process, which means that various pathogenic factors permeate the liver and lead to abnormal hyperplasia of connective tissue in the liver, which is a repair feedback mechanism for the body to deal with chronic liver injury [2]. Any liver injury has a process of liver fibrosis during the process of liver repair and healing. If the injury factors cannot be removed for a long time, the process of fibrosis will continue for a long time and develop into cirrhosis, even resulting in decompensated cirrhosis and various end-stage liver disease complications [3]. Related studies have shown that the early detection and symptomatic treatment of liver fibrosis symptoms can avoid the further development of liver fibrosis; otherwise, advanced cirrhosis is irreversible. Therefore, early diagnosis and symptomatic treatment of liver fibrosis are crucial [4]. 
Routine blood test is a common clinical non-invasive diagnosis method, which mainly includes HA, PIII NP, LN, and other indicators, but these indicators have low specificity for the diagnosis of liver fibrosis and can only be used as an auxiliary means [5]. In recent years, with the development of e-health imaging, SWE based on ultrasound technology has gradually become a new type of liver fibrosis diagnosis [6]. SWE can directly measure the Young's modulus value or shear wave velocity of liver tissue to judge the degree of tissue fibrosis classification, which has the advantages of quantitative data, non-invasiveness, and easy operation [7]. In addition, real-time analysis software can also be used to form elastic images on the basis of twodimensional ultrasound images, and different colors are introduced to display the distribution of elastic modulus in specific tissue regions. Jiang et al. [8] compared shear wave elastography and transient elastography to assess the accuracy of liver fibrosis staging in patients with non-alcoholic fatty liver disease. The AUC areas of early fibrosis, late fibrosis, and liver cirrhosis were $0.86,0.94$, and 0.95 , respectively, and the performance was excellent. Park et al. [9] analyzed the effects of shear wave elastography and serum aspartate aminotransferase on the detection of liver fibrosis and found that the diagnostic sensitivity and specificity of shear wave elasticity imaging for fibrosis and liver cirrhosis were significantly higher than those of serum aspartate aminotransferase. Therefore, the e-health-based SWE technology is currently a hot research topic.

To sum up, there are many diagnostic methods for the degree of liver fibrosis, and each has its advantages and disadvantages. The real-time SWE based on e-health is a new diagnostic method developed in recent years, but it has not been widely used in clinical practice. In this study, 73 patients with chronic HBV infection from August 2016 to November 2019 were selected as subjects for e-health SWE examination and serum liver fibrosis examination. Pearson correlation analysis was used to explore the correlation between elastic modulus values of different segments of the liver and four tests of serum hepatic fibrosis. ROC was used to analyze the sensitivity, specificity, and area under the curve (AUC) of SWE technical diagnosis to comprehensively evaluate the application value of SWE technology in the diagnosis of liver fibrosis in patients with chronic HBV infection.

\section{Materials and Methods}

2.1. Basic Information. 73 patients with chronic HBV infection who were admitted to hospital from August 2015 to November 2016 were selected as the study subjects, and they were divided into chronic hepatitis B group (51 cases) and hepatitis B cirrhosis group (22 cases). There were 36 males and 37 females, aged 40-85 years, with an average age of $57.96 \pm 7.92$ years, and another 20 healthy volunteers were introduced as control. After admission, all the patients underwent liver function, virology, and liver fibrosis examination on the 2nd day, and all the included subjects underwent e-health real-time shear wave elastic ultrasonic examination on the same day. This study had been approved by the medical ethics committee of the hospital. The patients and their families knew the advantages and disadvantages of participating in this study and were willing to cooperate with all kinds of tests required in the study plan and sign the informed consent.

2.1.1. Inclusion criteria. The diagnosis conformed to the criteria of The Guideline of Prevention and Treatment for Chronic Hepatitis B (updated version 2015) [10]: patients without other virus infection; patients without clinical treatment before testing; patients who have clear consciousness and can cooperate with the patient to complete the examination.

2.1.2. Exclusion criteria. The exclusion criteria were as follows: patients with liver parasite infection, cardiopulmonary, and other organ lesions; patients with incomplete imaging and clinical data; patients with abnormal cognition and poor compliance; patients who had received chemotherapy and radiotherapy; and patients who had taken liverprotecting enzyme lowering drugs.

2.2. Diagnosis and Index Detection Methods. The healthy population was taken as the control group, and the patients were all tested for liver function, hepatitis B two-and-half, serum liver fibrosis, and HBV-DNA.

Detection of HBV-DNA in serum was performed by fluorescence quantitative polymerase chain reaction (PCR). The main steps were as follows: taking the blood DNA of the patients as samples, $17.8 \mu \mathrm{L}$ of PCR reaction solution, $0.2 \mu \mathrm{L}$ of Tag enzyme, $0.02 \mu \mathrm{L}$ of UNG, and $2 \mu \mathrm{L}$ of sample solution were added to the PCR tube, respectively. The mixture was put into the PCR instrument, and the PCR program was set for detection. When $\mathrm{HBV}-\mathrm{DNA}>100 \mathrm{IU} / \mathrm{mL}$, it was positive.

Four tests of serum hepatic fibrosis including HA, LN, PIII NP, and CIV detection were performed. Enzymatic chemiluminescence immunoassay was mainly used for detection. Contents were detected in accordance with the instructions of the detection kit. The normal value ranges of the four tests were as follows: HA was within $0 \sim 100 \mathrm{ng} / \mathrm{mL}$, LN was within $0 \sim 50 \mathrm{ng} / \mathrm{mL}$, PIII NP was within $0 \sim 30 \mathrm{ng} / \mathrm{mL}$, and CIV was within $0 \sim 30 \mathrm{ng} / \mathrm{mL}$.

Liver function test: $1 \mathrm{~mL}$ blood samples were collected from all patients under fasting state, and the liver function was measured by automatic biochemical analyzer (Conas501).

Two halves of hepatitis B were quantitatively detected by time-resolved fluoroimmunoassay (TRFIA). When HBsAg was more than $0.5 \mathrm{ng} / \mathrm{mL}$, HBsAb was more than $10 \mathrm{mIU} /$ $\mathrm{mL}, \mathrm{HBeAb}$ was more than $0.2 \mathrm{PEIU} / \mathrm{mL}$, and $\mathrm{HBcAb}$ was more than $0.9 \mathrm{PEIU} / \mathrm{mL}$, it was positive.

2.3. Determination of Elastic Modulus of Liver. SuperSonic Imagine's Aixplorer instrument was used in all the included patients. SC6-1 convex array probe was adopted. The probe frequency was $3.5-5.5 \mathrm{MHz}$. The patient 
should lie on his back and raise his right leg. SWE examination was performed on the liver of the patient at an appropriate incision in the right intercostal section, and the elastic modulus was measured in the S2 and S3 segments of the left outer lobe of the liver, S4 segment of the left inner lobe of the liver, S5 and S8 segments of the right anterior lobe of the liver, and S6 and S7 segments of the right posterior lobe of the liver, avoiding the intrahepatic duct structure. The patient was advised to hold his breath for $3-5 \mathrm{~s}$. If the color filled the elastic sampling box above $90 \%$ and remained stable, the sampling was successful. Then, the elastic modulus of the liver in the imaging area was quantitatively measured. The system automatically calculated the mean, maximum, minimum, and standard deviation of the elastic modulus in the test area. When measuring, it was necessary to pay attention to adjust the elastic range according to the color display of the image. The range should be increased when the image was red and decreased when the color was light. The whole measurement was repeated 6 times.

2.4. Statistical Analysis. SPSS19.0 software was used for statistical analysis. The counting data were expressed as mean \pm standard error $(\bar{x} \pm s)$, LSD-t test was used for comparison between groups, and the classified data were compared using $\chi^{2}$. Pearson correlation test was used to detect the relationship between the results of liver fibrosis and the elastic modulus. Finally, ROC was used to analyze the sensitivity, specificity, and AUC area of SWE technology for patients with different fibrosis stages. $P<0.05$ was considered as a statistically significant difference.

\section{Results}

3.1. General Data Analysis of Patients. The differences in general data between the chronic hepatitis $\mathrm{B}$ group, the hepatitis B cirrhosis group, and the normal control group were compared, as shown in Table 1 . There was no significant difference in age, gender, and BMI between the chronic hepatitis B group, the hepatitis B cirrhosis group, and the normal control group $(P>0.05)$, while there was no significant difference in infection time between the chronic hepatitis B group and the hepatitis B cirrhosis group $(P>0.05)$.

3.2. Laboratory Test Results of Patients. The differences of HBV-DNA level, blood biochemical indexes, and serum hepatic fibrosis in the chronic hepatitis B group and the cirrhosis group were compared. As shown in Figure 1(a), after $\log$ conversion of HBV-DNA values in the two groups of patients, HBV-DNA content in the blood of the two groups of patients was $3.41 \pm 2.14 \mathrm{IU} / \mathrm{mL}$ and $2.98 \pm 3.62 \mathrm{IU} /$ $\mathrm{mL}$, respectively, and the difference between the two groups was not statistically significant $(P>0.05)$. The content of ALT and AST in the blood of the chronic hepatitis B group was significantly higher than that of the liver cirrhosis group $(102.43 \pm 65.12$ vs $91.06 \pm 45.62, \quad 113.43 \pm 44.13$ vs $98.21 \pm 60.72)(P<0.05)$. As shown in Figure $1(b)$, except for LN, the rest of the group of patients with chronic hepatitis B in serum HA, PIII NP, and CIV content were significantly lower than the second liver cirrhosis group $(100.23 \pm 63.84 \mathrm{vs}$ $412.67 \pm 106.76,24.34 \pm 15.68$ vs $72.39 \pm 35.33,40.74 \pm 20.28$ vs $102.34 \pm 40.37)(P<0.05)$.

3.3. Comparison of Patient SWE Collection Results. The success rate of SWE collection was compared between the chronic hepatitis B group, the hepatitis B cirrhosis group, and the normal control group, as shown in Table 2. It can be concluded that the detection success rate of S5 and S6 segments was $100.00 \%$, followed by that of S3 and S4 segments, while the acquisition success rate of S2, S7, and S8 segments was lower, and the difference between different groups in the acquisition success rate of each segment was statistically significant $(P<0.05)$.

Then, S3, S4, S5, and S6 sections with high acquisition success rate were selected to compare the differences between elastic modulus values. The results are shown in Figure 2. The elastic modulus values of the chronic hepatitis $\mathrm{B}$ group and the hepatitis B cirrhosis group at S3, S4, S5, and S6 were significantly higher than that of the normal control group, and the difference was statistically significant $(P<0.05)$; however, the elastic modulus values of the cirrhosis group at $\mathrm{S} 3, \mathrm{~S} 4, \mathrm{~S} 5$, and S6 were significantly higher than that of the chronic hepatitis $B$ group, and the difference was statistically significant $(P<0.05)$.

Then, real-time shear wave elastic ultrasonic diagnosis results of patients with different degrees of disease were compared, as shown in Figure 3. Ultrasound images of patients with mild chronic HBV infection showed that the liver echo was more uniform, the capsule was smooth, and the lower edge was slightly dull. As shown in Figure 3(b), ultrasound images of patients with moderate chronic HBV infection showed enhanced liver echo, relatively smooth capsule, and small tubercle protrusion.

3.4. Correlation Analysis of Elastic Modulus and Serum Hepatic Fibrosis in Patients. The correlations between the elastic modulus values of S5 and S6 segments in the chronic hepatitis B group, the hepatitis B cirrhosis group, and the serum hepatic fibrosis were compared respectively, as shown in Table 3. The elastic modulus value in S5 and S6 segments of chronic hepatitis B patients and HA, PIII NP, and CIV all had correlation, the $r$ value in S5 segment showed HA $>$ III $\mathrm{NP}=\mathrm{CIV}>\mathrm{LN}(0.51>0.50=0.50>0.25)$, and the $r$ value in S6 segment showed $\mathrm{HA}>\mathrm{CIV}>\mathrm{PIII} \mathrm{NP}>\mathrm{LN}$ $(0.53>0.52>0.47>0.26)$. The elastic modulus value in S5 and $\mathrm{S} 6$ segments of the hepatitis B cirrhosis patients and HA, PIII NP, and CIV all have correlation, and $r$ value in S5 segment showed $\mathrm{HA}>\mathrm{CIV}>\mathrm{PIII} \quad \mathrm{NP}>\mathrm{LN}$ $(0.79>0.70>0.52>0.24)$, and $r$ value in S6 segment showed $\mathrm{HA}>\mathrm{CIV}>\mathrm{PIII} \mathrm{NP}>\mathrm{LN} \quad(0.81<0.65>0.54>0.22)$. The one with the highest $r$ value was selected to display the correlation scatter graph, and the results are shown in Figures 4(a) and 4(b). The elastic modulus values of S5 and S6 segments were positively correlated with HA content in patients with hepatitis B cirrhosis. 
TABLE 1: General data analysis of patients.

\begin{tabular}{lccr}
\hline Projects & $\begin{array}{c}\text { The chronic hepatitis B group } \\
(n=51)\end{array}$ & $\begin{array}{c}\text { The hepatitis B cirrhosis group } \\
(n=22)\end{array}$ & $\begin{array}{c}\text { The normal control group } \\
(n=20)\end{array}$ \\
\hline Age (year) & $35.86 \pm 13.18$ & $40.81 \pm 11.09$ & $36.92 \pm 4.17$ \\
Male (\%) & $32(62.75 \%)$ & $17(77.27 \%)$ & $13(65.00 \%)$ \\
BMI index $\left(\mathrm{kg} / \mathrm{m}^{2}\right)$ & $21.67 \pm 2.36$ & $22.06 \pm 2.54$ & $P>0.05$ \\
Infection time (year) & $6.52 \pm 4.19$ & $5.97 \pm 3.27$ & $21.07 \pm 2.30$ \\
\hline
\end{tabular}

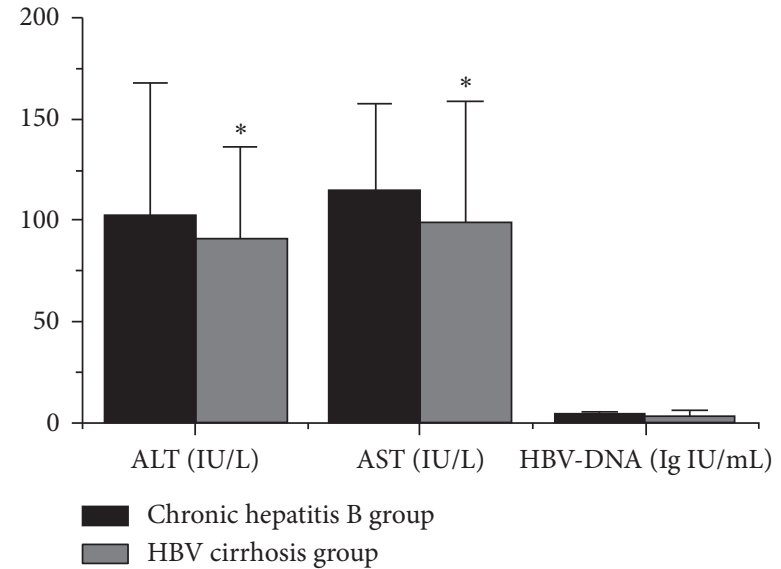

(a)

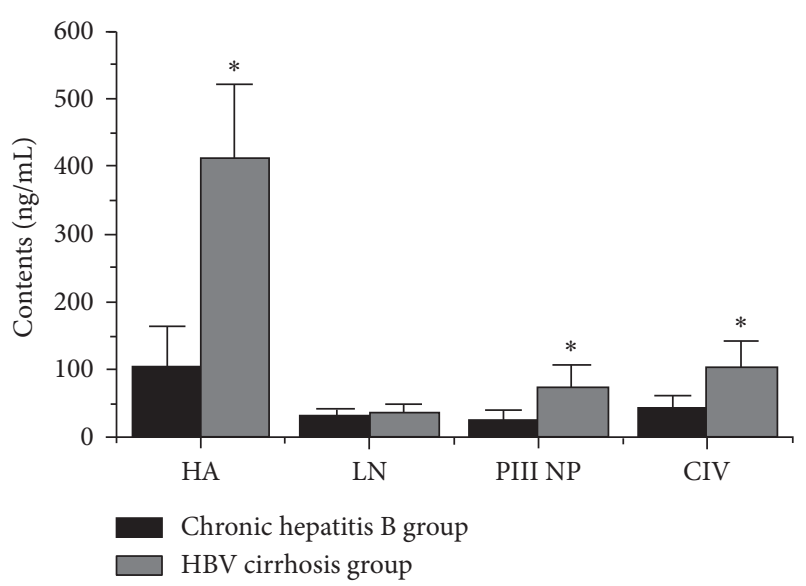

(b)

Figure 1: Comparison of laboratory results of patients. Note. (a) The test results of HBV-DNA level, ALT, and AST. (b) The four test results of liver fibrosis; * represents compared with the chronic hepatitis B group, the difference was statistically significant $(P<0.05)$.

TABLE 2: The success rate of SWE collection for each liver segment in each group.

\begin{tabular}{|c|c|c|c|}
\hline The liver segment & The chronic hepatitis B group $(n=51)$ & The hepatitis B cirrhosis group $(n=22)$ & The normal control group $(n=20)$ \\
\hline S2 & $0(0.00 \%)$ & $2(9.09 \%)$ & $0(0.00 \%)$ \\
\hline S3 & $34(66.67 \%)$ & $17(77.27 \%)$ & $10(50.00 \%)$ \\
\hline S4 & $22(43.14 \%)$ & $14(63.64 \%)$ & $9(45.00 \%)$ \\
\hline S5 & $51(100.00 \%)$ & $22(100.00 \%)$ & $0(0.00 \%)$ \\
\hline S6 & $51(100.00 \%)$ & $22(100.00 \%)$ & $0(0.00 \%)$ \\
\hline S7 & $1(1.96 \%)$ & $3(13.64 \%)$ & $0(0.00 \%)$ \\
\hline S8 & $4(7.84 \%)$ & $0(0.00 \%)$ & $1(5.00 \%)$ \\
\hline$\chi^{2}$ value & 431.52 & 97.26 & 103.94 \\
\hline$P$ value & $P<0.01$ & $P<0.01$ & $P<0.01$ \\
\hline
\end{tabular}

3.5. The ROC Curve Analysis of Fibrosis Degree in Patients with Chronic Liver Disease through SWE Diagnosis. As shown in Figure 5, sensitivity, specificity, and AUC area of fibrosis degree in SWE patients diagnosed with chronic liver disease were analyzed using the subject operating curve. The AUC area was 0.866 , the sensitivity was $89.59 \%$, and the specificity was $76.49 \%$.

\section{Discussion}

Liver biopsy has always been regarded as the gold standard for diagnosing the degree of liver fibrosis. Unfortunately, this technique has certain limitations, such as the risk of trauma patients, poor repeatability, and susceptibility to subjective influence of doctors. Therefore, it is urgent to find a new method to diagnose liver fibrosis [11]. Four tests of serum hepatic fibrosis are widely used in clinical practice, namely, HA, LN, PIII NP, and CIV. Transient elastography is a new dispute technique based on ultrasound that has emerged in recent years. It is mainly a system that can be measured by shear elasticity detector by utilizing the correlation between liver fibrosis and liver elasticity. The accuracy and sensitivity of its diagnosis in the degree of hepatic fibrosis have been proved by many studies. Therefore, SWE technology based on e-health was used in this study to measure the liver elastic modulus of patients to quantitatively show the degree of liver fibrosis of patients. Moreover, after testing different segments of the liver, it was found that the success rate of S5 and S6 segments was 100\%, while the success rate of S7 and S8 segments was relatively low, which was different from the research results of Perry et al. [12]. The reason may be that S7 and S8 were located deeper than 


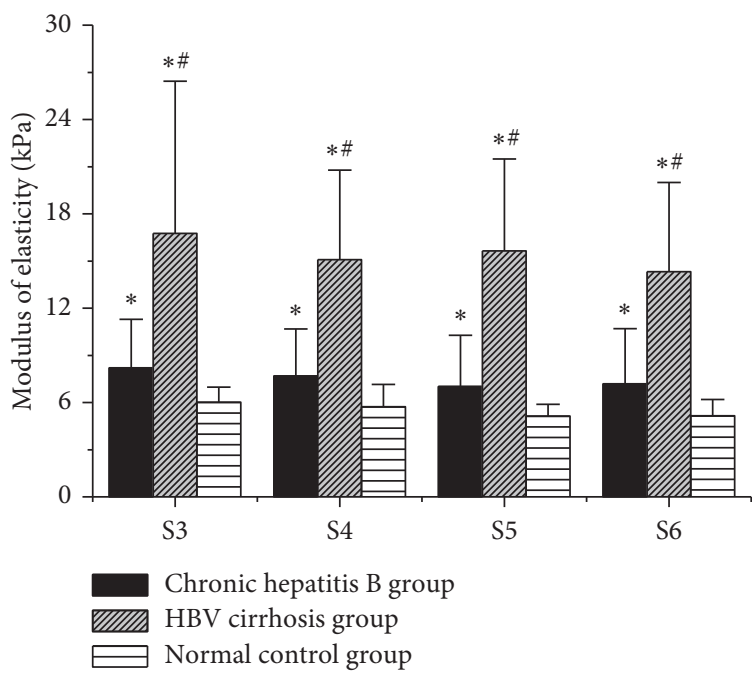

Figure 2: Results of the elastic modulus values of the liver of the patients. Note. $*$ indicates statistically significant difference compared with the control group $(P<0.05)$; \# indicates that compared with the chronic hepatitis B group the difference was statistically significant $(P<0.05)$.

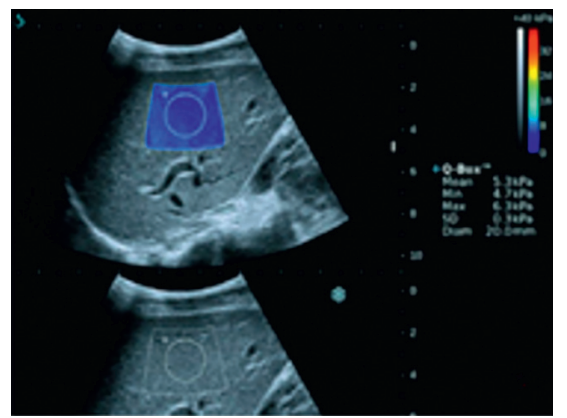

(a)

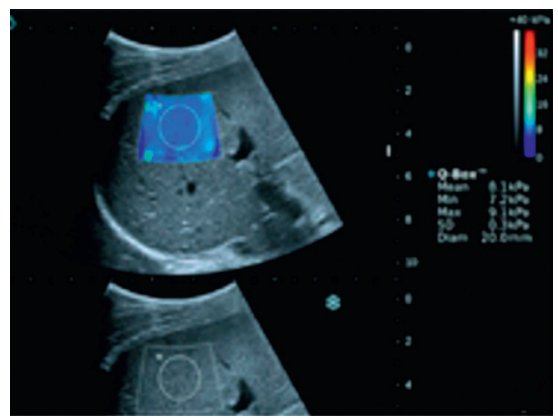

(b)

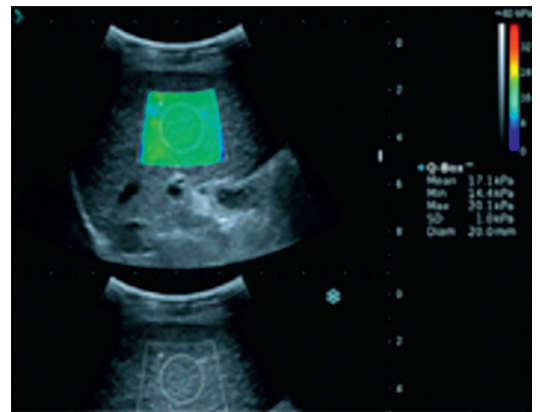

(c)

Figure 3: Partial images of liver of real-time shear wave elastic ultrasound diagnosis. (a) Normal control group; (b) the liver manifestations of patients with mild chronic HBV; (c) the liver manifestations of patients with moderate and chronic HBV.

TABle 3: The correlation between elastic modulus values of S5 and S6 segments and serum hepatic fibrosis in patients.

\begin{tabular}{|c|c|c|c|c|c|c|}
\hline Grouping & The liver segments & & $\mathrm{HA}$ & LN & PIII NP & $\mathrm{CIV}$ \\
\hline & S5 & $\begin{array}{c}r \\
P \text { value }\end{array}$ & $\begin{array}{c}0.51 \\
P<0.01\end{array}$ & $\begin{array}{c}0.25 \\
P<0.01\end{array}$ & $\begin{array}{c}0.50 \\
P<0.01\end{array}$ & $\begin{array}{c}0.50 \\
P<0.01\end{array}$ \\
\hline Ihe chronic hepattis B group & S6 & $\begin{array}{c}\mathrm{r} \\
P \text { value }\end{array}$ & $\begin{array}{c}0.53 \\
P<0.01\end{array}$ & $\begin{array}{c}0.26 \\
P<0.01\end{array}$ & $\begin{array}{c}0.47 \\
P<0.01\end{array}$ & $\begin{array}{c}0.52 \\
P<0.01\end{array}$ \\
\hline The hepatitis B cirrhosis group & S6 & $\begin{array}{c}\mathrm{r} \\
P \text { value } \\
\mathrm{r} \\
P \text { value }\end{array}$ & $\begin{array}{c}0.79 \\
P<0.01 \\
0.81 \\
P<0.01\end{array}$ & $\begin{array}{c}0.24 \\
P<0.01 \\
0.22 \\
P<0.01\end{array}$ & $\begin{array}{c}0.52 \\
P<0.01 \\
0.54 \\
P<0.01\end{array}$ & $\begin{array}{c}0.70 \\
P<0.01 \\
0.65 \\
P<0.01\end{array}$ \\
\hline
\end{tabular}

S5 and S6 and easily affected by the beating of the heart, so the detection success rate was very low, which indicated that S5 and S6 segments should be selected preferentially when SWE was used to detect the elasticity of patients with liver lesions to improve the detection efficiency.

Chronic HBV viral hepatitis is usually accompanied by liver fibrosis of varying degrees. Because of the persistent presence of the virus, repeated or continuous inflammatory infiltration can lead to pathological changes such as inflammation and necrosis in liver parenchyma, resulting in continuous fibrous hyperplasia of the liver and the formation of hepatic fibrosis [13]. Studies have shown that the level of CIV increased significantly in the serum of patients with chronic active hepatitis, cirrhosis, and hepatocellular carcinoma [14]. And many studies have shown that HA is an important marker for the level of liver fibrosis. In addition, HA can also reflect the degree of liver damage [15]. However, it was later found that the values of $\mathrm{HA}$ and CIV in patients 


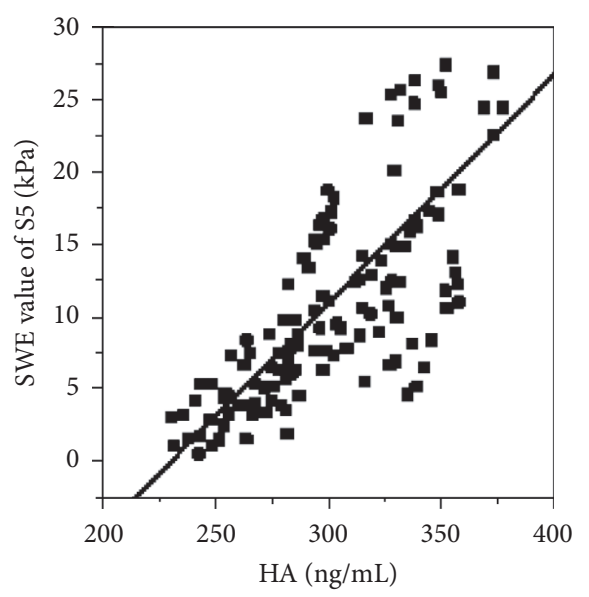

(a)

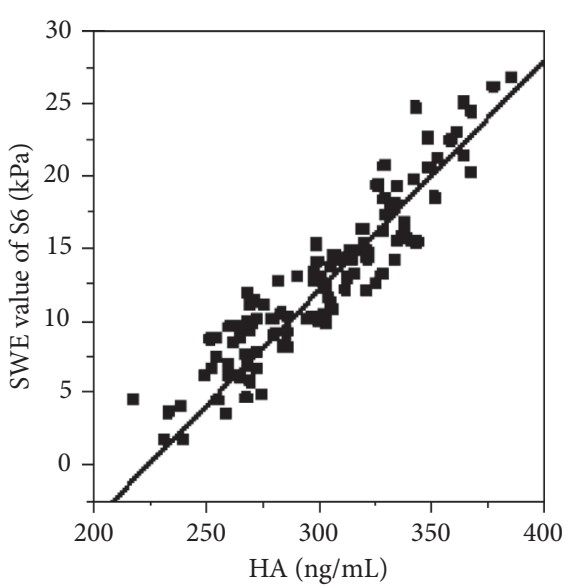

(b)

Figure 4: The scatter plot of correlation between elastic values of S5 and S6 segments and HA in patients with hepatitis B cirrhosis. Note. (a) The correlation of segment S5 $(r=0.79, P<0.01)$. (b) The correlation of segment S6 $(r=0.81, P<0.01)$.

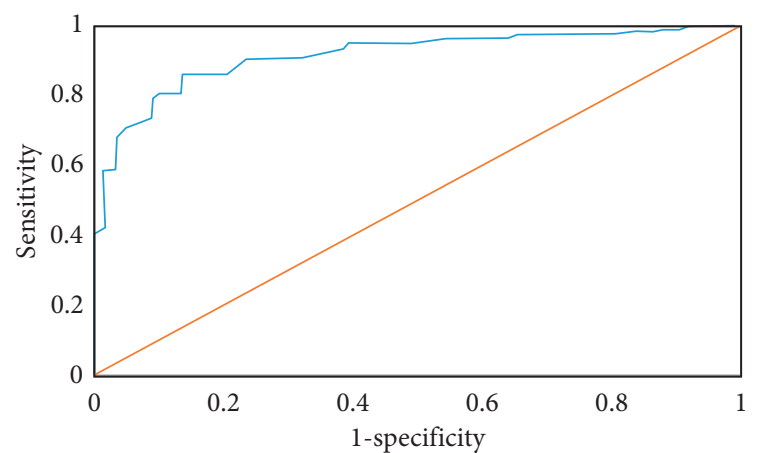

FIGURE 5: The ROC curve for the SWE diagnosis of fibrosis degree in patients.

with cirrhosis B were highly correlated with the elastic modulus values in S5 and S6 segments, indicating that the changes in HA and CIV contents should be given priority when observing the changes in the degree of fibrosis in patients. Other studies have shown that LN mainly reflects hepatic fibrosis and portal vein pressure, but it was found in the study that the correlation of LN was lowest compared to the other three indicators. It was found in the study that the correlation between elastic modulus and HA in patients with different segments of hepatitis B cirrhosis was higher than that in patients with chronic hepatitis B, indicating that the severer the disease, the higher the degree of liver fibrosis [16]. In addition, the sensitivity, specificity, and AUC area of SWE in diagnosing fibrosis in patients with chronic liver disease were analyzed using the subject operating curve. The results showed that the AUC area was 0.866 , the sensitivity was $89.59 \%$, and the specificity was $76.49 \%$, which is consistent with the results of $77 \%$ sensitivity and $79 \%$ specificity obtained by Deurdulian et al. [17] in 147 American SWE tests on the degree of liver fibrosis in 111 adult liver transplantation patients. It also has higher sensitivity, indicating SWE's reliability in diagnosing fibrosis in patients with chronic liver disease.

\section{Conclusion}

The application value of real-time shear wave elastography based on e-health in the diagnosis of liver fibrosis in patients with chronic HBV infection was quantitatively analyzed by detecting liver elastic modulus value. However, there are still some shortcomings in this study. The sample size of selected patients is relatively small, and the source is single. Most of the samples of patients are from outpatient clinics, no biopsy is performed for pathological typing, and SWE analysis of patients with liver fibrosis and inflammation at different stages will be conducted in combination with pathological test results for future research. It is necessary to increase the selection of patient samples for further research. In conclusion, the liver elastic modulus value measured by real-time shear wave elastography based on e-health can quantitatively show liver fibrosis in patients with chronic HBV infection and has a high sensitivity, so its application in the diagnosis of clinical liver fibrosis degree is real and feasible.

\section{Data Availability}

No data were used to support this study.

\section{Conflicts of Interest}

The authors declare that they have no conflicts of interest.

\section{References}

[1] L. Yang, F. Liu, X. Tong, D. Hoffmann, J. Zuo, and M. Lu, "Treatment of chronic hepatitis B virus infection using small molecule modulators of nucleocapsid assembly: recent advances and perspectives," ACS Infectious Diseases, vol. 5, no. 5, pp. 713-724, 2019.

[2] M. Sohrabi, M. N. Tosi, P. Azar et al., "The impact of transient elastography in the assessment and follow-up of patients with HBeAg-negative chronic hepatitis B virus infection," Govaresh, vol. 24, no. 2, pp. 111-117, 2019. 
[3] S.-S. Ji, H.-D. Jiang, J.-C. Jiang et al., "Applicability of liver stiffness measurement based nomograms to the assessments of hepatitis B related significant fibrosis and cirrhosis," Clinica Chimica Acta, vol. 489, pp. 75-82, 2019.

[4] V. Lo Re, C. W. Newcomb, D. M. Carbonari et al., "Determinants of liver complications a HIV/hepatitis B virus-c patients," JAIDS Journal of Acquired Immune Deficiency Syndromes, vol. 82, no. 1, pp. 71-80, 2019.

[5] Y. Zhuang, H. Ding, Y. Zhang, H. Sun, C. Xu, and W. Wang, "Two-dimensional shear-wave elastography performance in the noninvasive evaluation of liver fibrosis in patients with chronic hepatitis B: comparison with serum fibrosis indexes," Radiology, vol. 283, no. 3, pp. 873-882, 2017.

[6] H. Maruyama, K. Kobayashi, S. Kiyono, T. Sekimoto, T. Kanda, and O. Yokosuka, "Two-dimensional shear wave elastography with propagation-based reliability assessment for grading hepatic fibrosis and portal hypertension," Journal of Hepato-Biliary-Pancreatic Sciences, vol. 23, no. 9, pp. 595-602, 2016.

[7] E. S. Lee, J. B. Lee, H. R. Park et al., "Shear wave liver elastography with a propagation map: diagnostic performance and inter-observer correlation for hepatic fibrosis in chronic hepatitis," Ultrasound in Medicine and Biology, vol. 43, no. 7, pp. 1355-1363, 2017.

[8] W. Jiang, S. Huang, H Teng et al., "Diagnostic accuracy of point shear wave elastography and transient elastography for staging hepatic fibrosis in patients with non-alcoholic fatty liver disease: a meta-analysis," BMJ Open, vol. 8, no. 8, Article ID e021787, 2018.

[9] D. W. Park, Y. J. Lee, W Chang et al., "Diagnostic performance of a point shear wave elastography ( $\mathrm{pSWE}$ ) for hepatic fibrosis in patients with autoimmune liver disease," PLoS One, vol. 14, no. 3, 2019.

[10] Chinese Society of Hepatology, Chinese Medical Association, "The guideline of prevention and treatment for chronic hepatitis B: a 2015 update," Journal of Clinical Hepatology, vol. 31, no. 12, pp. 1941-1960, 2015.

[11] B. Hu, W. Huang, R. Wang et al., "High rate of detection of human ESPL1-HBV $S$ fusion gene in patients with HBVrelated liver cancer: a Chinese case-control study," Anticancer Research, vol. 40, no. 1, pp. 245-252, 2020.

[12] M. T. Perry, N. Savjani, E. I. Bluth, A. Dornelles, and G. Therapondos, "Point shear wave elastography in assessment of hepatic fibrosis," Ultrasound Quarterly, vol. 32, no. 3, pp. 201-207, 2016.

[13] D. Zarębska-Michaluk, A. Piekarska, J. Jaroszewicz et al., "Comparative effectiveness of 8 versus 12 weeks of ombitasvir/ paritaprevir/ritonavir and dasabuvir in treatment-naïve patients infected with $\mathrm{HCV}$ genotype $1 \mathrm{~b}$ with non-advanced hepatic fibrosis," Advances in Medical Sciences, vol. 65, no. 1, pp. 12-17, 2020.

[14] X. L. Wei, "The relationship between serum visfatin and the progress of chronic viral hepatitis B cirrhosis," European Review for Medical and Pharmacological Sciences, vol. 21, no. 2, pp. 297-301, 2017.

[15] R. ń Ingvar, P. Peter, and L. Stefan, "Diagnostic accuracy of $\alpha 1$-acid glycoprotein fucosylation for liver cirrhosis in patients undergoing hepatic biopsy," Clinical Chemistry, vol. 48, no. 12, pp. 2195-2201, 2002.

[16] Y.-c. Jiang, Y.-f. Li, L. Zhou, and D.-p. Zhang, "Comparative metabolomics unveils molecular changes and metabolic networks of syringin against hepatitis B mice by untargeted mass spectrometry," RSC Advances, vol. 10, no. 1, pp. 461-473, 2020.
[17] C. Deurdulian, E. G. Grant, H. Tchelepi et al., "Assessment of fibrosis in liver transplant recipients: diagnostic performance of shear wave elastography (SWE) and correlation of SWE findings with biopsy results," American Journal of Roentgenology, vol. 213, no. 6, pp. W264-W271, 2019. 